\title{
MAXIMAL SUBSETS OF PAIRWISE NONCOMMUTING ELEMENTS OF SOME $p$-GROUPS OF MAXIMAL CLASS
}

\author{
S. FOULADI and R. ORFI ${ }^{凶}$
}

(Received 13 February 2011)

\begin{abstract}
Let $G$ be a group. A subset $X$ of $G$ is a set of pairwise noncommuting elements if $x y \neq y x$ for any two distinct elements $x$ and $y$ in $X$. If $|X| \geq|Y|$ for any other set of pairwise noncommuting elements $Y$ in $G$, then $X$ is said to be a maximal subset of pairwise noncommuting elements. In this paper we determine the cardinality of a maximal subset of pairwise noncommuting elements for some $p$-groups of maximal class. Specifically, we determine this cardinality for all 2-groups and 3-groups of maximal class.
\end{abstract}

2010 Mathematics subject classification: primary 20D15; secondary 20D60.

Keywords and phrases: p-groups of maximal class, $A C$-group.

\section{Introduction}

Let $G$ be a nonabelian group and let $X$ be a maximal subset of pairwise noncommuting elements of $G$. The cardinality of such a subset is denoted by $\omega(G)$. Also $\omega(G)$ is the maximal clique size in the noncommuting graph of a group $G$. Let $Z(G)$ be the center of $G$. The noncommuting graph of a group $G$ is a graph with $G \backslash Z(G)$ as the vertices and join all distinct vertices $x$ and $y$ such that $x y \neq y x$. By a famous result of Neumann [8], answering a question of Erdős, the finiteness of $\omega(G)$ in $G$ is equivalent to the finiteness of the factor group $G / Z(G)$. Chin [4] has obtained upper and lower bounds for $\omega(G)$ for an extra-special $p$-group $G$, where $p$ is an odd prime number. For $p=2$, it has been shown by Isaacs (see [3, p. 40]) that $\omega(G)=2 n+1$ for any extra-special group $G$ of order $2^{2 n+1}$. In [1, 2], $\omega(\operatorname{GL}(n, q))$ is given for $n \in\{2,3\}$.

Let $G$ be a $p$-group of maximal class and order $p^{n}$, where $p$ is a prime number. In this paper we find $\omega(G)$ when $G$ satisfies some extra conditions. On the other hand, for these groups of maximal class we show that $\omega(G)=p^{n-2}+1$ or $\omega(G)=$ $p^{n-2}+p+1$ (Theorems 3.4 and 3.7). Then by the above observation we determine $\omega(G)$ for all 2-groups and 3-groups $G$ of maximal class. In particular, we show that $\omega(G)=2^{n-2}+1$ for any 2-group $G$ of maximal class and order $2^{n}$ (Corollary 3.10). Also for a 3-group $G$ of maximal class and order $3^{n}$ we show that $\omega(G)=3^{n-2}+1$

(C) 2011 Australian Mathematical Publishing Association Inc. 0004-9727/2011 \$16.00 
when $G$ possesses an abelian maximal subgroup, and $\omega(G)=3^{n-2}+4$ when $G$ possesses no abelian maximal subgroups (Corollary 3.11).

Throughout this paper we use the following notation. The letter $p$ denotes a prime number. The terms of the lower central series of $G$ are denoted by $\gamma_{i}=\gamma_{i}(G)$. A group $G$ is called an $A C$-group if the centralizer of every noncentral element of $G$ is abelian and $\mathcal{C}_{G}(x)$ is the centralizer of an element $x$ in a group $G$.

\section{Some basic results}

In this section we give some basic results that are needed for the main results of the paper.

LEMMA 2.1. The following conditions on a group $G$ are equivalent:

(i) $G$ is an AC-group;

(ii) if $[x, y]=1$ then $\mathcal{C}_{G}(x)=\mathcal{C}_{G}(y)$, where $x, y \in G \backslash Z(G)$.

PROOF. This is straightforward. See also [9, Lemma 3.2].

LEMMA 2.2. Let $G$ be an AC-group.

(i) If $a, b \in G \backslash Z(G)$ with distinct centralizers, then $\mathcal{C}_{G}(a) \cap \mathcal{C}_{G}(b)=Z(G)$.

(ii) If $G=\bigcup_{i=1}^{k} \mathcal{C}_{G}\left(a_{i}\right)$, where $\mathcal{C}_{G}\left(a_{i}\right)$ and $\mathcal{C}_{G}\left(a_{j}\right)$ are distinct for $1 \leq i<j \leq k$, then $\left\{a_{1} \cdots a_{k}\right\}$ is a maximal set of pairwise noncommuting elements in $G$.

Proof. (i) We see that $Z(G) \leq \mathcal{C}_{G}(a) \cap \mathcal{C}_{G}(b)$. If $Z(G)<\mathcal{C}_{G}(a) \cap \mathcal{C}_{G}(b)$, then there exists an element $x$ in $\mathcal{C}_{G}(a) \cap \mathcal{C}_{G}(b)$ such that $x \notin Z(G)$. This means that $\mathcal{C}_{G}(a)=\mathcal{C}_{G}(x)$ and $\mathcal{C}_{G}(b)=\mathcal{C}_{G}(x)$ by Lemma 2.1(ii), which is impossible.

(ii) By Lemma 2.1(ii), $\left\{a_{1}, a_{2}, \ldots, a_{k}\right\}$ is a set of pairwise noncommuting elements. Suppose to the contrary that $\left\{b_{1}, b_{2}, \ldots, b_{t}\right\}$ is another set of noncommuting elements of $G$ with $t>k$. Then we see that there exist positive integers $r, s$ and $i$ with $1 \leq r<s \leq t$ and $1 \leq i \leq k$ such that $b_{r}, b_{s} \in \mathcal{C}_{G}\left(a_{i}\right)$. This yields $\mathcal{C}_{G}\left(b_{r}\right)=$ $\mathcal{C}_{G}\left(b_{s}\right)$ by Lemma 2.1(ii), or equivalently $b_{r} b_{s}=b_{s} b_{r}$, which is a contradiction.

LEMMA 2.3. Let $G$ be a finite group of order $p^{n}$ with the central quotient of order $p^{2}$, where $p$ is a prime number. Then $\omega(G)=p+1$.

Proof. First we show that $G$ is an $A C$-group. Suppose that $a$ is a noncentral element of $G$. So $Z(G)<\mathcal{C}_{G}(a)$. Therefore $\left|\mathcal{C}_{G}(a)\right|=p^{n-1}$. Since $\mathcal{C}_{G}(a)=\langle Z(G), a\rangle$, we see that $\mathcal{C}_{G}(a)$ is abelian and so $G$ is an $A C$-group. Now since $G$ is finite we may write $G=\bigcup_{i=1}^{k} \mathcal{C}_{G}\left(a_{i}\right)$, where $\mathcal{C}_{G}\left(a_{i}\right)$ and $\mathcal{C}_{G}\left(a_{j}\right)$ are distinct for $1 \leq i<j \leq k$. Therefore $X=\left\{a_{1}, a_{2}, \ldots, a_{k}\right\}$ is a maximal subset of pairwise noncommuting elements of $G$ by Lemma 2.2(ii). Thus by Lemma 2.2(i),

$$
|G|=\sum_{i=1}^{k}\left(\left|\mathcal{C}_{G}\left(a_{i}\right)\right|-|Z(G)|\right)+|Z(G)| .
$$

This yields $p^{n}=k \times\left(p^{n-1}-p^{n-2}\right)+p^{n-2}$ and so $k=p+1$. 


\section{Main results}

Let $G$ be a $p$-group of maximal class and order $p^{n}(n \geq 4)$, where $p$ is a prime. Following [7], we define the 2-step centralizer $K_{i}$ in $G$ to be the centralizer in $G$ of $\gamma_{i}(G) / \gamma_{i+2}(G)$ for $2 \leq i \leq n-2$ and define $P_{i}=P_{i}(G)$ by $P_{0}=G, P_{1}=K_{2}$, $P_{i}=\gamma_{i}(G)$ for $2 \leq i \leq n$. The degree of commutativity $l=l(G)$ of $G$ is defined to be the maximum integer such that $\left[P_{i}, P_{j}\right] \leq P_{i+j+l}$ for all $i, j \geq 1$ if $P_{1}$ is not abelian and $l=n-3$ if $P_{1}$ is abelian.

In this section we determine $\omega(G)$ for any $p$-group $G$ of maximal class and order $p^{n}$, with positive degree of commutativity when $\left[P_{1}, P_{3}\right]=1$. Then we deduce $\omega(G)$ for all 2-groups and 3-groups of maximal class

LEMMA 3.1. Let $G$ be a p-group of maximal class which possesses an abelian maximal subgroup. Then $P_{1}$ is abelian.

Proof. Let $M$ be an abelian maximal subgroup of $G$. Then $\left[M, \gamma_{2}(G)\right]=1$. This implies that $P_{1}=M$ by using the definition of $P_{1}$.

Lemma 3.2 [7, Corollary 3.2.7]. Let $G$ be a p-group of maximal class. The degree of commutativity of $G$ is positive if and only if the 2-step centralizers of $G$ are all equal.

THEOREM 3.3. Let $G$ be a p-group of maximal class and order $p^{n}(n \geq 4)$ with positive degree of commutativity and let $s \in G \backslash P_{1}, s_{1} \in P_{1} \backslash P_{2}$ and $s_{i}=\left[s_{i-1}, s\right]$ for $1 \leq i \leq n-1$. Then:

(i) $G=\left\langle s, s_{1}\right\rangle, P_{i}=\left\langle s_{i}, \ldots, s_{n-1}\right\rangle,\left|P_{i}\right|=p^{n-i}$ for $1 \leq i \leq n-1$ and $P_{n-1}(G)=$ $Z(G)$ is of order $p$;

(ii) $\mathcal{C}_{G}(s)=\langle s\rangle P_{n-1}, s^{p} \in P_{n-1}$ and $\left|\mathcal{C}_{G}(s)\right|=p^{2}$. So

$$
\mathcal{C}_{G}(s)=\left\{s^{i} s_{n-1}^{j}: 0 \leq i, j \leq p-1\right\}
$$

(iii) $\mathcal{C}_{G}(s) \cap\left(G \backslash P_{1}\right)=\left\{s^{i} s_{n-1}^{j}: 1 \leq i \leq p-1,0 \leq j \leq p-1\right\}$;

(iv) $\mathcal{C}_{G}(s) \cap P_{1}=Z(G)$;

(v) if $s, s^{\prime} \in G \backslash P_{1}$ and $\left[s, s^{\prime}\right] \neq 1$, then $\mathcal{C}_{G}(s) \cap \mathcal{C}_{G}\left(s^{\prime}\right)=Z(G)$.

PROOF. (i) This is obvious by [7, Lemma 3.2.4].

(ii) This follows from Lemma 3.2 and [6, Hilfssatz III 14.13].

(iii) and (iv) are evident.

(v) We have $Z(G) \leq \mathcal{C}_{G}(s) \cap \mathcal{C}_{G}\left(s^{\prime}\right)<\mathcal{C}_{G}(s)$. Also by (i) and (ii), we see that $|Z(G)|=p$ and $\left|\mathcal{C}_{G}(s)\right|=p^{2}$ which completes the proof.

THEOREM 3.4. Let $G$ be a p-group of maximal class and order $p^{n}(n \geq 4)$ with positive degree of commutativity which possesses an abelian maximal subgroup. Then:

(i) $G$ is an AC-group;

(ii) $\omega(G)=p^{n-2}+1$.

Proof. (i) By Lemma 3.1, $P_{1}$ is abelian and so $\mathcal{C}_{G}(x)=P_{1}$ for any $x \in P_{1} \backslash Z(G)$. Moreover, if $x \in G \backslash P_{1}$, then by Theorem 3.3(ii), $\left|\mathcal{C}_{G}(x)\right|=p^{2}$ as desired. 
(ii) We may write $G=\bigcup_{i=1}^{m} \mathcal{C}_{G}\left(a_{i}\right)$, where $\mathcal{C}_{G}\left(a_{i}\right)$ and $\mathcal{C}_{G}\left(a_{i}\right)$ are distinct for $1 \leq i<j<m$ and $a_{i} \notin Z(G)$. Therefore $\left\{a_{1}, \ldots, a_{m}\right\}$ is a maximal subset of pairwise noncommuting elements of $G$ by Lemma 2.2(ii). Now let $s_{1} \in P_{1} \backslash P_{2}$ as in Theorem 3.3. Then we may assume that $s_{1} \in \mathcal{C}_{G}\left(a_{1}\right)$ and so $\mathcal{C}_{G}\left(s_{1}\right)=\mathcal{C}_{G}\left(a_{1}\right)$ by Lemma 2.1. Moreover, $\mathcal{C}_{G}\left(s_{1}\right)=P_{1}$ by considering the proof of (i). Therefore $\left|\mathcal{C}_{G}\left(a_{1}\right)\right|=\left|P_{1}\right|=p^{n-1}$. So $a_{i} \notin P_{1}$ for $2 \leq i \leq m$. Therefore $\left|\mathcal{C}_{G}\left(a_{i}\right)\right|=p^{2}$ for $2 \leq i \leq m$ by Theorem 3.3(ii). On the other hand,

$$
|G|=\left|\mathcal{C}_{G}\left(a_{1}\right)\right|+\sum_{i=2}^{m}\left(\left|\mathcal{C}_{G}\left(a_{i}\right)\right|-|Z(G)|\right)
$$

by Lemma 2.2(i). This means that $p^{n}=p^{n-1}+(m-1)\left(p^{2}-p\right)$ which yields $\omega(G)=p^{n-2}+1$.

LEMMA 3.5. Let $G$ be a p-group of maximal class and order $p^{n}(n \geq 4)$ with positive degree of commutativity and $\left[P_{1}, P_{3}\right]=1$ such that $G$ possesses no abelian maximal subgroup. Then $P_{1}$ is not abelian and $\omega\left(P_{1}\right)=p+1$.

Proof. Note that $P_{1}$ is not abelian since $P_{1}$ is a maximal subgroup of $G$. Also, $P_{3} \leq$ $Z\left(P_{1}\right)$ by the fact that $\left[P_{1}, P_{3}\right]=1$. Therefore $\left|P_{1} / Z\left(P_{1}\right)\right|=p^{2}$ by Theorem 3.3(i). Now the result follows from Lemma 2.3.

LEMMA 3.6. By the assumption of Lemma 3.5, if $Y=\left\{y_{1}, \ldots y_{t}\right\}$ is a maximal subset of pairwise noncommuting elements in $G \backslash P_{1}$, then $t=p^{n-2}$.

Proof. On setting $A_{i}=\mathcal{C}_{G}\left(y_{i}\right) \cap\left(G \backslash P_{1}\right)$ for $1 \leq i \leq t$, we see that $\left|A_{i}\right|=$ $p(p-1)$ and $A_{i} \cap A_{j}=\emptyset$ for $1 \leq i<j \leq t$ by Theorem 3.3(iii), (v). We claim that $G \backslash P_{1}=A_{1} \cup \cdots \cup A_{t}$. For otherwise if $y \in G \backslash P_{1}$ and $y \notin A_{i}$ for $1 \leq i \leq t$, then $y \notin \mathcal{C}_{G}\left(y_{i}\right)$. This implies that $\left\{y, y_{1}, \ldots, y_{t}\right\}$ is a subset of pairwise noncommuting elements in $G \backslash P_{1}$, which is a contradiction. Therefore $G \backslash P_{1}=A_{1} \cup \cdots \cup A_{t}$ is a partition for $G \backslash P_{1}$. Hence $\left|G \backslash P_{1}\right|=\sum_{i=1}^{t}\left|A_{i}\right|$ and so $p^{n}-p^{n-1}=p(p-1) t$, and consequently $t=p^{n-2}$.

THEOREM 3.7. Let $G$ be a p-group of maximal class and order $p^{n}(n \geq 4)$ with positive degree of commutativity and $\left[P_{1}, P_{3}\right]=1$ such that $G$ possesses no abelian maximal subgroup. Then $\omega(G)=p^{n-2}+p+1$.

Proof. Let $Y=\left\{y_{1}, \ldots, y_{t}\right\}$ and $X=\left\{x_{1}, \ldots, x_{p+1}\right\}$ be maximal subsets of pairwise noncommuting elements in $G \backslash P_{1}$ and $P_{1}$, respectively. Obviously, by Lemma 3.6, $t=p^{n-2}$. First we see that $\left[x_{i}, y_{j}\right] \neq 1$ for $1 \leq i \leq p+1$ and $1 \leq j \leq t$. For otherwise $x_{i} \in \mathcal{C}_{G}\left(y_{j}\right)$ and so $x_{i} \in \mathcal{C}_{G}\left(y_{j}\right) \cap P_{1}$ or, equivalently, $x_{i} \in Z(G)$ by Theorem 3.3(iv), which is impossible. Therefore $X \cup Y$ is a subset of pairwise noncommuting elements in $G$ and so $\omega(G) \geq t+p+1=p^{n-2}+p+1$. Now let $\omega(G)=m$ and $\left\{a_{1}, \ldots, a_{m}\right\}$ be a maximal subset of pairwise noncommuting elements in $G$. We may assume that $\left\{a_{1}, \ldots, a_{k}\right\} \subseteq G \backslash P_{1}$ and $\left\{a_{k+1}, \ldots, a_{m}\right\} \subseteq P_{1}$. By Lemma 3.5, we see that $m-k \leq \omega\left(P_{1}\right)=p+1$. Also, by Lemma 3.6, we have $k \leq p^{n-2}$. Consequently $\omega(G)=m-k+k \leq p+1+p^{n-2}$ as desired. 
Now we determine $\omega(G)$ for all 2-groups and 3-groups $G$ of maximal class by using the following two theorems.

THEOREM 3.8 [7, Theorem 3.4.1]. Let $G$ be a 2-group of maximal class. Then $P_{1}$ is cyclic.

THEOREM 3.9 [7, Theorem 3.4.3]. Let $G$ be a 3-group of maximal class. Then $G$ has degree of commutativity $l \geq n-4$.

COROLlaRY 3.10. If $G$ is a 2-group of maximal class and order $2^{n}$, then $\omega(G)=$ $2^{n-2}+1$.

ProOf. This is evident for $n=3$ by Lemma 2.3. Now since $P_{1}$ is abelian and $n \geq 4$, the degree of commutativity of $G$ is $n-3$. Therefore we can complete the proof by Theorem 3.4.

COROllary 3.11. Let $G$ be a 3-group of maximal class and order $3^{n}$.

(i) If $G$ possesses an abelian maximal subgroup, then $\omega(G)=3^{n-2}+1$.

(ii) If $G$ possesses no abelian maximal subgroup, then $\omega(G)=3^{n-2}+4$.

ProOf. This is obvious for $n=3$ by Lemma 2.3. Also for $n=4$, we see that $\omega(G)=10$ by using GAP [5]. Now we may assume that $n \geq 5$. Moreover, $G$ has degree of commutativity $l \geq n-4$ by Theorem 3.9 and so $\left[P_{1}, P_{3}\right]=1$. Therefore we can complete the proof by using Theorems 3.4 and 3.7.

\section{References}

[1] A. Abdollahi, A. Akbari and H. R. Maimani, 'Non-commuting graph of a group', J. Algbera 298(2) (2006), 468-492.

[2] A. Azad and C. E. Praeger, 'Maximal subsets of pairwise non-commuting elements of threedimensional general linear groups', Bull. Aust. Math. Soc. 80(1) (2009), 91-104.

[3] E. A. Bertram, 'Some applications of graph theory to finite groups', Discrete Math. 44(1) (1983), $31-43$.

[4] A. M. Y. Chin, 'On non-commuting sets in an extraspecial p-group', J. Group Theory 8(2) (2005), 189-194.

[5] The GAP Group, 'GAP - Groups, Algorithms, and Programming', Version 4.4.12, 2008, http://www.gap-system.org.

[6] B. Huppert, Endliche Gruppen, I (Springer, Berlin, 1967).

[7] C. R. Leedham-Green and S. McKay, The Structure of Groups of Prime Power Order, London Mathematical Society Monographs, New Series, 27 (Oxford University Press, Oxford, 2002).

[8] B. H. Neumann, 'A problem of Paul Erdős on groups', J. Aust. Math. Soc. Ser. A 21(4) (1976), 467-472.

[9] D. M. Rocke, 'p-groups with abelian centralizers', Proc. Lond. Math. Soc. 30(3) (1975), 55-57.

S. FOULADI, Department of Mathematics, University of Arak, Arak, Iran e-mail: s-fouladi@araku.ac.ir

R. ORFI, Department of Mathematics, University of Arak, Arak, Iran e-mail: r-orfi@araku.ac.ir 